\title{
Polar lipids: n-3 PUFA carriers for membranes and brain: nutritional interest and emerging processes
}

\author{
Michel PARMENTIER \\ C. AL SAYED MAHMOUD \\ Michel LINDER \\ Jacques FANNI \\ Nancy-Université, \\ INPL-ENSAIA, \\ Laboratoire de Science et Cénie Alimentaires, \\ Vandouvre-les-Nancy, France
}

\begin{abstract}
The $n-3$ fatty acids are unanimously considered as high nutritional value molecules, especially Long-Chains PUFA from marine origin. However, most of the products available in the market contain LC-PUFA esterified on the glycerol under the common form of triacylglycerols. Another interesting way can be to esterify the PUFA on polar lipids and especially on Phospholipids. An original patented enzymatic process carried out under mild conditions (low temperature, no use of organic solvent) is presented to produce at industrial scale a Phospho-Lipo-Peptidic Complex, that is particularly rich in DHA esterified on the sn-2 position on PL.
\end{abstract}

Key words: phospholipids, n-3 polyunsaturated fatty acids, enzymatic processing
The nutritional interest of the $n-3$ fatty acids in terms of prevention of cardiovascular and other ageing diseases has been extensively studied under numbered animal and clinical human trials. There is some evidence that the intake of long-chain n-3 polyunsaturated fatty acids (LC-PUFA) may influence the status of lipids, particularly the fatty acids composition in blood, cell membranes or different organs and compartments of the human body. A recent study in rats carried out in relation with ITERG, Pessac, France, on margarines supplemented by LC-PUFA vs. precursors (Linoleic and Alpha-Linolenic Acids) clearly demonstrated a strong relationship between the diet and the PUFA content of blood lipids, cholesterol esters and membrane phospholipids of the erythrocytes [1].

\section{Precursors vs. LC-PUFA, available sources}

Only few studies are dealing with the molecular form of PUFA intake, since a majority of natural or supplemented products are constituted by triacylglycerols in which the PUFA are esterified under their final chemical form. Another basic question regarding the PUFA intake is related to the type of PUFA: should we consider precursors vs. long chain PUFA? Starting from $\alpha$-Linolenic Acid, the liver of the mammalians is able to produce $\omega 3$-LC-PUFA, mainly EPA and DHA through the action of a complex enzymatic pool of desaturases and elongases [2]. However,

- EPA is both an important terminal molecule and the substrate for the elongation and desaturation that lead to DHA. Therefore, the yield is generally weak for this final molecule of the 13 series, especially in the human metabolism.

- The same enzymatic pool is involved in the metabolism of Linoleic Acid that generates the $\omega 6$-LC-PUFA series, one of the main terminal molecules being Arachidonic Acid (ARA). For instance, these $\omega 6$-PUFA display antagonist properties regarding inflammation. The competition between the substrates ALA/LA turns in favour of the $\omega 6$ series since the global intake ratio $\omega 6 / \omega 3$ is often higher than the recommended ratio, which would be close to 5 [3]. The conclusion is that the amounts of DHA available for the global body metabolism remain insufficient for a large part of the humans and an intake of dietary 103 -LC-PUFA is highly recommended. Because the only available source of $\omega 3$-LCPUFA comes from marine lipids for the moment, the best recommendation that can be given is to eat fish. A promising process to produce LC-PUFA using engineered oleaginous way is under investigation, but only preliminary results have been recently obtained and efficient vegetable systems are expected not before 10 years [4].

\section{Molecular form of PUFA intake in Food}

There are several molecular ways to carry fatty acids from diet to metabolism (table 1). The major molecular form is represented by triacylglycerols (TAG). In fact, $98 \%$ of the fatty acids come from TAG (50-100 $\mathrm{g}$ per day for an adult). Phospholipids (PL) are the second dietary source, mainly represented by lecithins (2-10 g per day). Apart from these two main sources,

other nutritional minor molecular species are also available, such as diacylglycerols (DAG) which are present as minor components in oils and fats (1-5 g per day), as well as cholesterol esters (CE) (0.15-0.4 $\mathrm{g}$ per day) and fat soluble vitamin esters, such as retinyl palmitate and tocopherol acetate $(1 \mathrm{mg}$ and $15 \mathrm{mg}$ per day, respectively).

Ethyl esters (FAEE) and free fatty acids (FFA) are possible alternative sources of bioavailable fatty

Table 1. From food intake to metabolism: Fatty acid vectors (Fave et al., 2004.






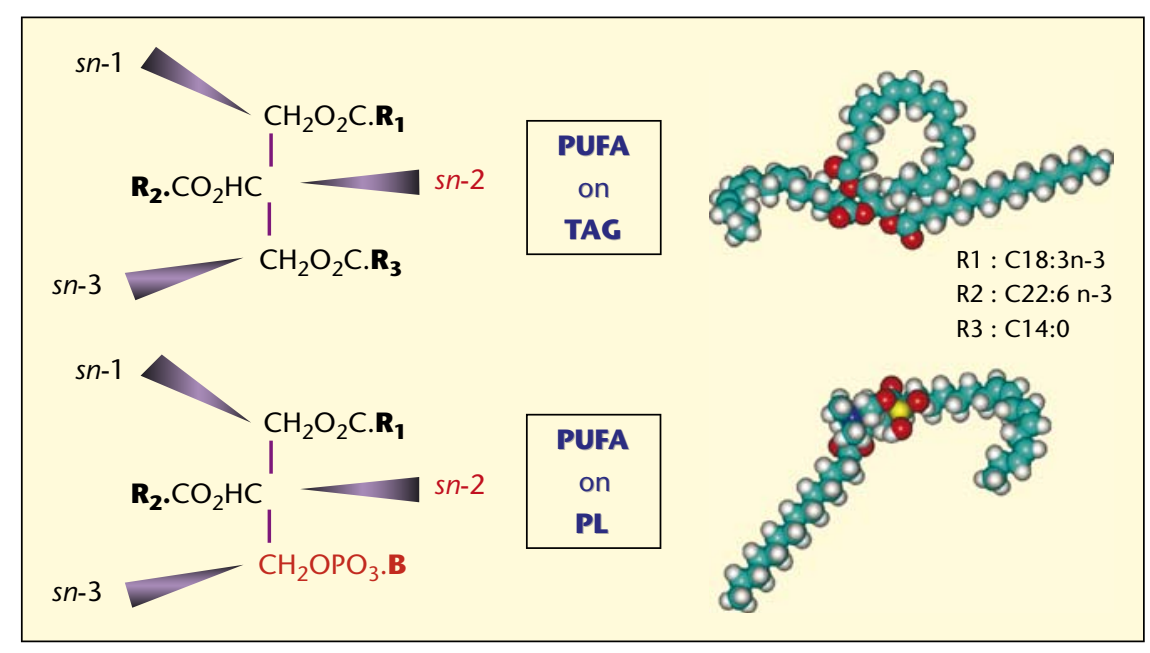

Figure 1. Comparative structure of TAG and PL.

acids but they are not naturally present in edible oils and fats.

Concerning the main sources that are TAG and $\mathrm{PL}$, it is well known that the bioavailability of a given fatty acid highly depends on 2 important parameters:

- Its ability to produce highly dispersed droplets into the liquid medium of the stomach. This property is required to obtain the best compatibility between the oily phases and water, to permit the lipases and other enzymes to be efficient.

- Its location on the glycerol backbone (figure 1): best bioavailability is obtained when the fatty acid is at the sn2 position, thus preserving it under 2-monoglyceride or 2-lysophospholipid forms during the intestinal assimilation process.

\section{Polar lipids vs. Triacylglycerols}

Furthermore, polar lipids and especially phospholipids (PL) could be another interesting way to carry LC-PUFA into the organism. This is particularly true when a PUFA is esterified at the sn2 position as observed in marine PL, especially from Salmon [5]. This property has not been as widely studied as in the triacylglycerol way, because products containing a large amount of PL with sn2-LC-PUFA are not commonly available in the market, except some lipidic fractions from fish or crustaceans extracted with organic solvents.

Phospholipids commonly occur in vegetable oils and animal fats, but they are still considered as impurities. Therefore, degumming is the first step of the refining process of vegetable oils. Residual amounts of PL in edible oils are less than 5 ppm (expressed in P content). However, their functional properties are com- the efficacy of PUFA supplementation was higher by using Phospholipids instead of TAC as established in a dose-response study [7]. These benefits could be due to the amphiphilic properties of the phospholipids reflected in a better water dispersibility and a greater susceptibility to phospholipases compared to the glycerolysis of TAG.

Several studies have concerned the brain function: a supplementation of DHA on PL improves behaviour, learning and visual functions in 13 -deficient mice, as demonstrated by Carrie et al., 2002 [8]. Moreover, in mice with PUFA deficiency, Bourre et al., 2002 showed that a PUFA on phospholipids supplementation was more efficient than a TAG form to recover a normal AG composition in brain [9]. A particular PL, phosphatidylcholine (PC), has been proposed to be a specific AG carrier to the brain by Lagarde et al., 2001 [10].

Other effects related to ageing have been investigated: Rotstein et al. (1987) have observed that DHA-rich PL decreased in the retina during ageing [11], as well as the turnover of the PL in the synaptic membranes decreased [12]. Bourre (1992) established that the efficacy of some hepatic desaturases decreased during ageing, this making worse the DHA potential deficiency [13].

\section{Nutritional interest of polar lipids}

The question is: is there any interest to use $\mathrm{PL}$ as vectors for PUFA rather than TAG? The particular interest of marine PL consists in a high degree of unsaturation, with ARA, EPA and DHA mainly esterified at the $s n-2$ position. Consequently, their intestinal absorption is high according to Bourre, 2004 [6]. This property has been confirmed by using a Caco- 2 cells assay (figure 2). The permeability of the PUFA on PL was found 5 times higher and the final intracellular level 50 times higher than PUFA on TAG. Wijendran et al. (2002) have shown that

\section{Phospholipids and brain}

Specific studies have concerned the effect of dietary PL on the brain functions. Kidd (1999) showed that PC improved memory and learning performances in ageing human [14]. In the same order, Bernoud et al. (1999) proposed Lyso-PC as the most efficient carrier of DHA to specific brain tissues [15]. Brain preferentially absorbs DHA as sn-2 Lyso-PC compared with unesterified DHA [16]. On the contrary, PS appeared to be concentrated in the cell membranes of the brain. A comparison between TAG- and PL-fed groups indicated that only PL

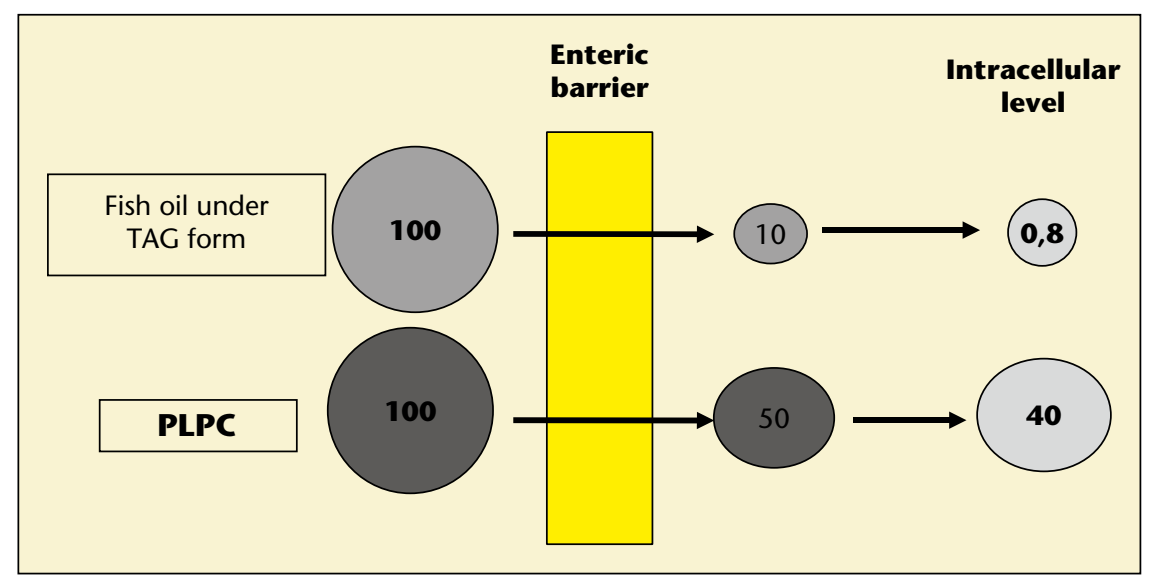

Figure 2. PLPC 13 -PUFA bioavailability: preliminary results on Caco-2 cells. 
supplementation increased brain $\mathrm{DHA}$, as established by Werner et al., 2004 [17]. Regarding brain diseases, PL have been found to have a beneficial effect in dementia, depression, and other diseases. Moreover, PL have been supposed to improve some brain functions like memory. However, all these global effects are still controversial, even though this has been claimed by some nutraceuticals for years.

\section{Extraction and enrichment processes}

Assuming that there is some interest to produce concentrated $\mathrm{PL}$, especially marine $\mathrm{PL}$ that are particularly rich in PUFA, what are the industrial available processes for extracting these molecules from natural complex stuffs? First of all, let's consider the available PUFA-rich phospholipids sources for production at industrial level. The term "marine lecithin" is used to describe a mixture of phospholipids (PC, PE, $\mathrm{PS}, \mathrm{PI}$...) obtained from a marine raw material, i.e. Krill and other crustaceans, roe and milt of fish such as salmon, herring, capelin and cod..., also marine by-products from the filleting industry.

All these products are rich in LC-PUFA phospholipids, depending on the species.

Pig or other mammalians brain extracts can be considered as good sources; unfortunately they have been disqualified by the recent BSE crisis for safety concerns. So, it can be assumed that marine PL are the only industrial source available in safe conditions.

Several patents are dealing with this challenge, but all the processes described are based on the extraction of phospholipids from lipid mixtures using organic solvents (acetone, methanol, propanol...):

- Products containing highly unsaturated fatty acids for use by women and their children during stages of preconception, pregnancy and lactation/post-partum [18].

- Method for extraction of a lipid mixture containing PUFA-rich PL from viscera of fish, method for preserving viscera prior to extraction, and lipid mixture extracted thereby [19]. - Extraction of phospholipids from fish roe by SC-CO2, following by 3 ethanol extraction steps [20] (figure 3).

- Extraction of phospholipids from Krill by successive acetone and alcohol treatments [21] (figure 4).

\section{A novel enzymatic extraction process (patented [22])}

As said above, all processes involve drastic chemical conditions. Such conditions are now

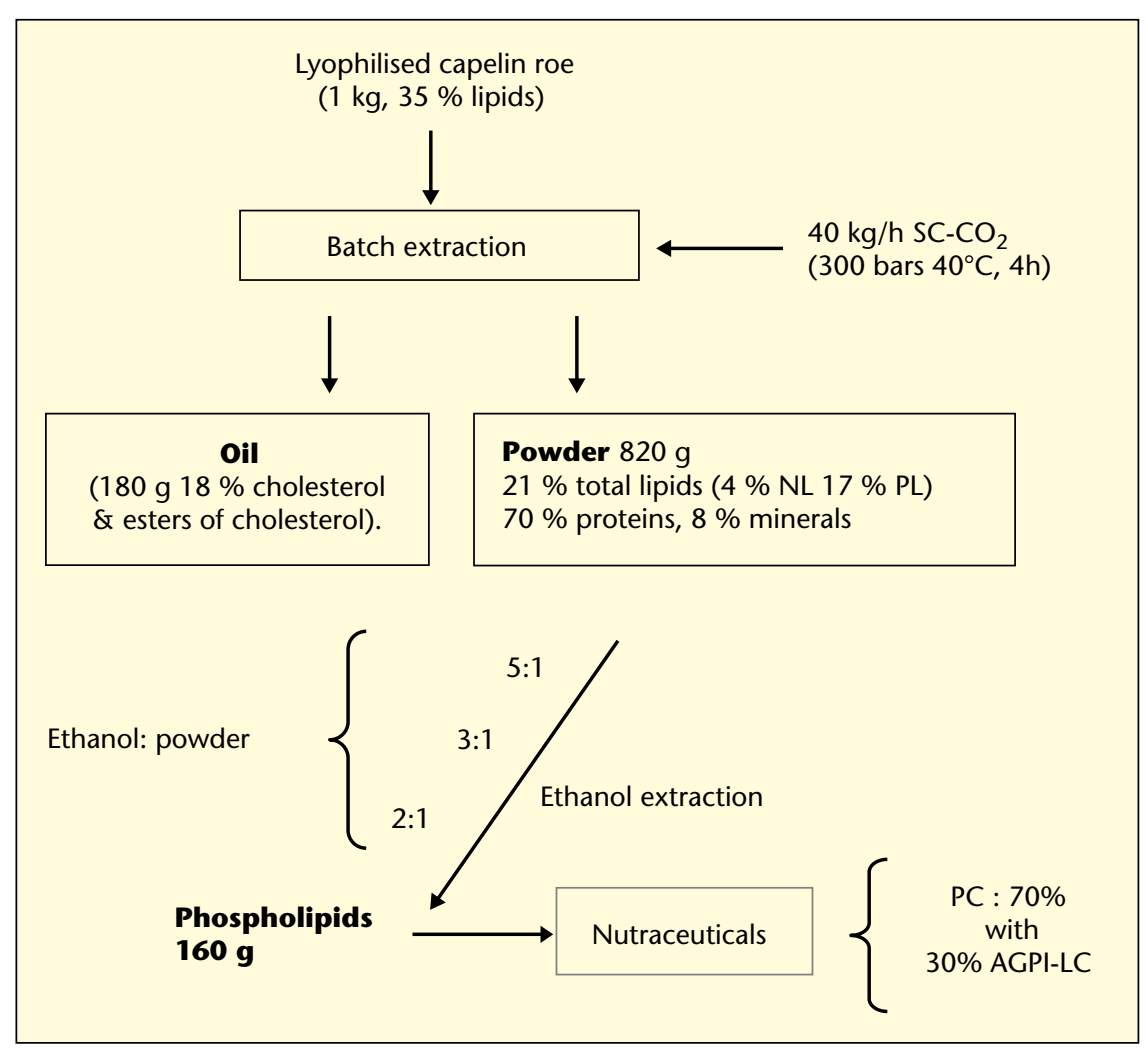

Figure 3. Extraction of phospholipids from fish roe (Leigh et al., 2004).

out of the scope of nutritional and nutraceutical products constraints. Therefore, looking for alternative mild processes, carried out at low temperature and solvent-free conditions was an interesting scientific and industrial challenge.

Starting from a natural source of LCPUFA esterified on PL, which is the brain tissue of Salmo salar, (see composition on table 2) we have investigated a mild process [22] without any use of organic solvent and able to provide an extract that is rich in $\mathrm{PL}$, itself being particularly rich in DHA (> 30\%). This enzymatic process provides at industrial level a product that have been called Phospho-Lipo-Peptidic Complex (PLPC) available for a nutraceutical use with the special target to deliver a high concentration of DHA on PL in the specific metabolic pathway of these molecules.
As described figure 5, heads of salmon are crushed in an adequate water amount and the mixture is submitted to the action of proteases. The enzymatic hydrolysis is monitored to achieve a destructuration of the animal tissue able to allow subsequent separation by centrifugation.

The products obtained are a soluble protein hydrolysate, a pure oily phase (neutral oil), and a mixture that contains phospholipids, peptides and oil. The FA-compositions of these different fractions are given table 3 . It is noticeable that the PLPC lipid phase is highly unsaturated, containing near to $40 \%$ of n-3 PUFA, with a ratio DHA/EPA reaching 2.16 . The $\mathrm{PL}$ phase of the PLPC contains more than $30 \%$ DHA with a ratio n-3/n-6 equal to 8.6 (figure 6). The study of the oily phases of the PLPC by TLC-FID, after development in hexane/diethyl

Table 2. Physico-chemical composition of a filleting by-product of Salmo salar.

\begin{tabular}{|lcc|}
\hline Composition & Head & Filet \\
\hline Proteins (\%) & $13.1 \pm 0.4$ & $18.2 \pm 0.5$ \\
Lipids (\%) & $23.8 \pm 0.5$ & $14.5 \pm 1.5$ \\
Dry mater (\%) & $67.8 \pm 1.3$ & $36.8 \pm 0.5$ \\
Ash (\%) & $2.6 \pm 0.6$ & $1.3 \pm 0.1$ \\
\hline
\end{tabular}




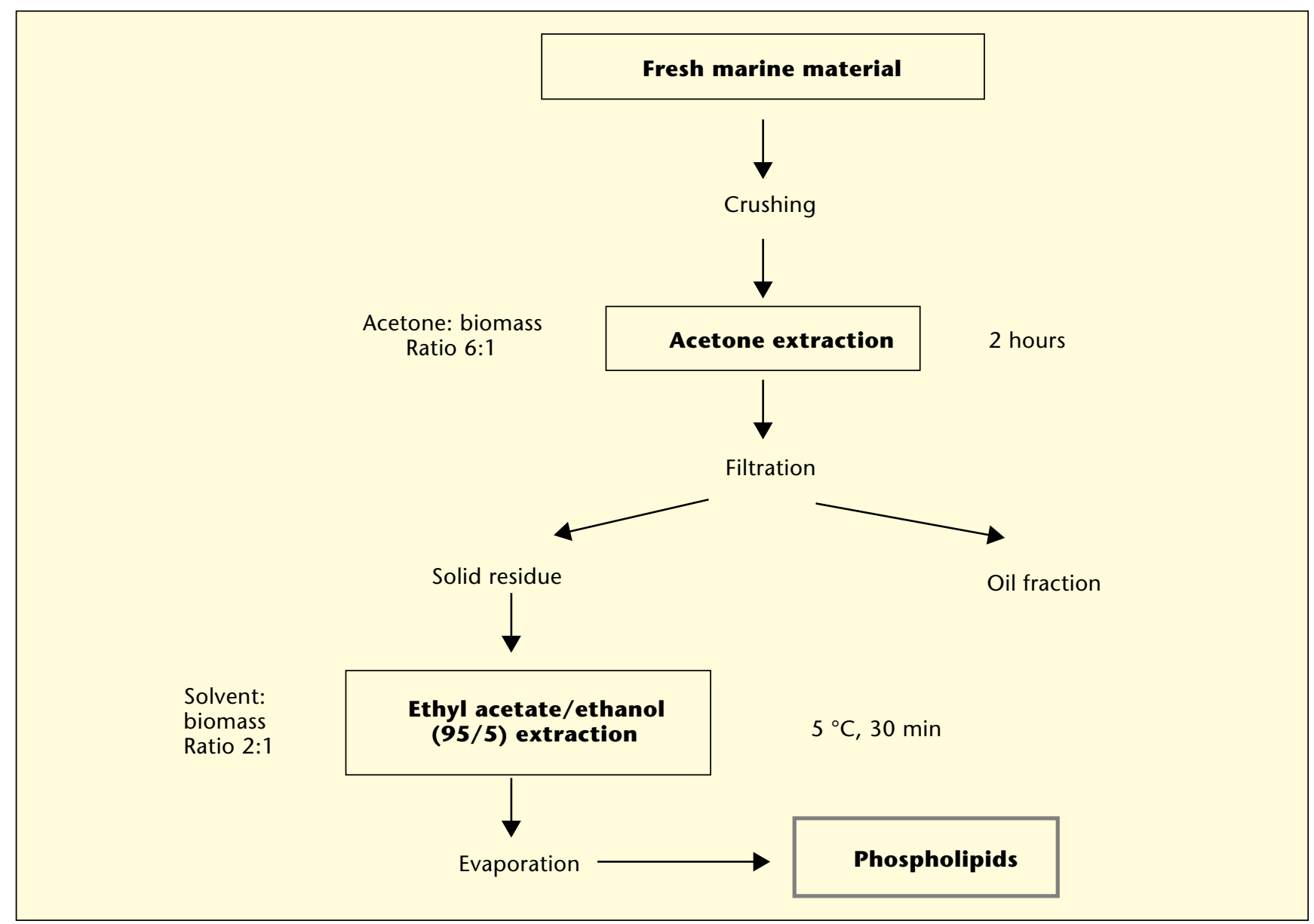

Figure 4. Extraction of phospholipids from Krill : successive acetone and alcohol treatments (Fotini Sampalis, 2004).

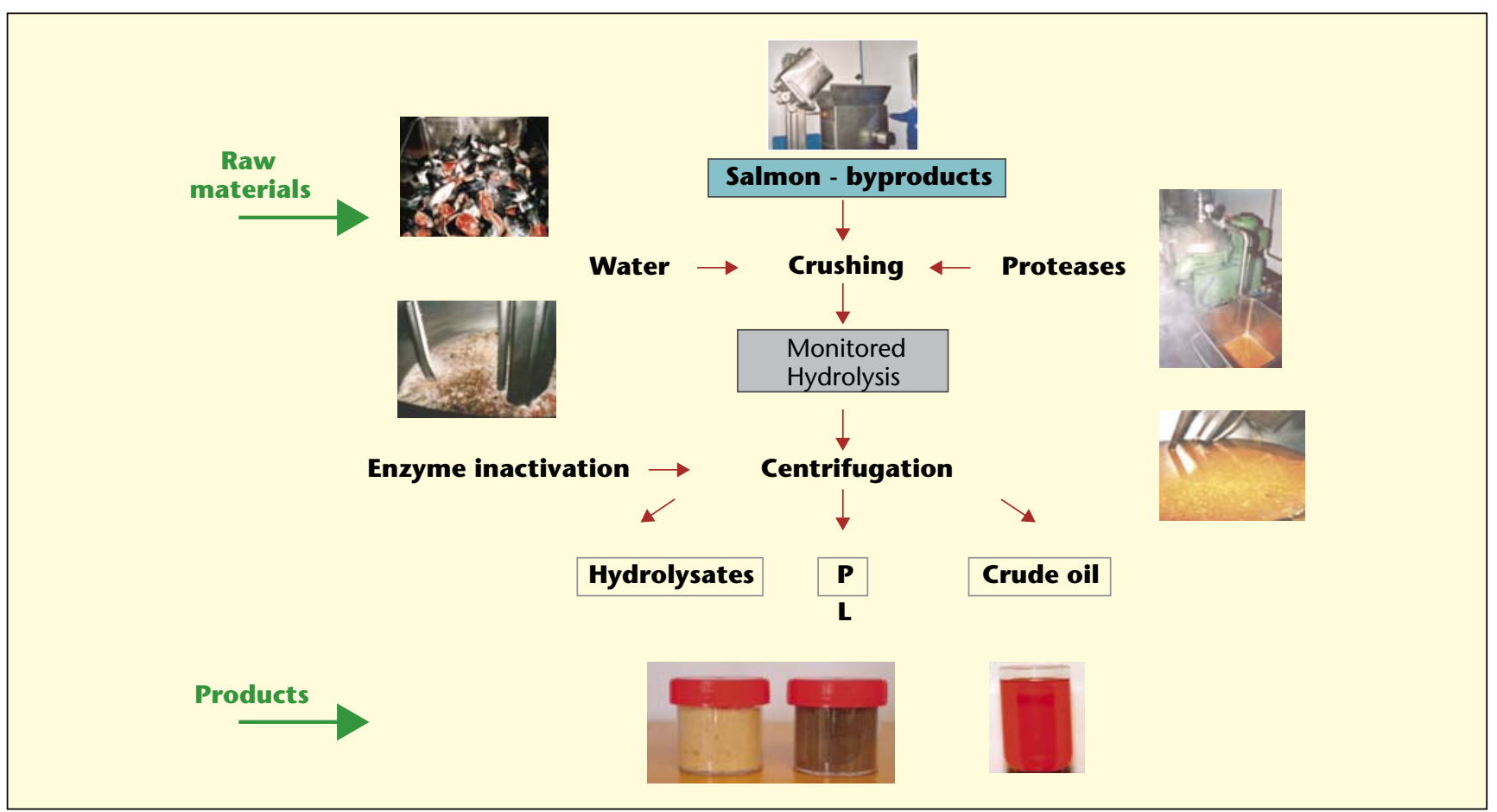

Figure 5. Marine lipid enzymatic extraction (low temperature, no use of organic solvent (Linder et al., 2002). 
Table 3. Fatty acid composition of the raw material and the fractions extracted from a salmon by-product after enzymatic hydrolysis (neutral oil, Phospho-lipo-peptidic complex (PLPC) and phospholipids (PL) of the PLPC.

\begin{tabular}{|c|c|c|c|c|}
\hline Fatty acids groups & Salmon heads (raw material) & Neutral oil & PLPC & PL of the PLPC \\
\hline$\sum$ SFA & 24.65 & 25.23 & 23.64 & 19.29 \\
\hline$\Sigma$ MUFA & 39.92 & 40.78 & 31.34 & 24.00 \\
\hline$\Sigma$ PUFA & 35.42 & 34.02 & 45.13 & 56.71 \\
\hline$\sum$ PUFA n-6 & 7.73 & 7.78 & 5.59 & 5.86 \\
\hline$\sum$ PUFA n-3 & 27.69 & 26.24 & 39.04 & 50.44 \\
\hline EPA & $8.43 \pm 0.18$ & $7.74 \pm 0.48$ & $8.19 \pm 0.14$ & $10.31 \pm 0.10$ \\
\hline DHA & $12.10 \pm 0.64$ & $11.90 \pm 0.70$ & $18.18 \pm 0.39$ & $33.10 \pm 0.40$ \\
\hline DHA/EPA & 1.44 & 1.54 & 2.16 & 3.44 \\
\hline$n-3 / n-6$ & 3.58 & 3.17 & 6.98 & 8.60 \\
\hline
\end{tabular}

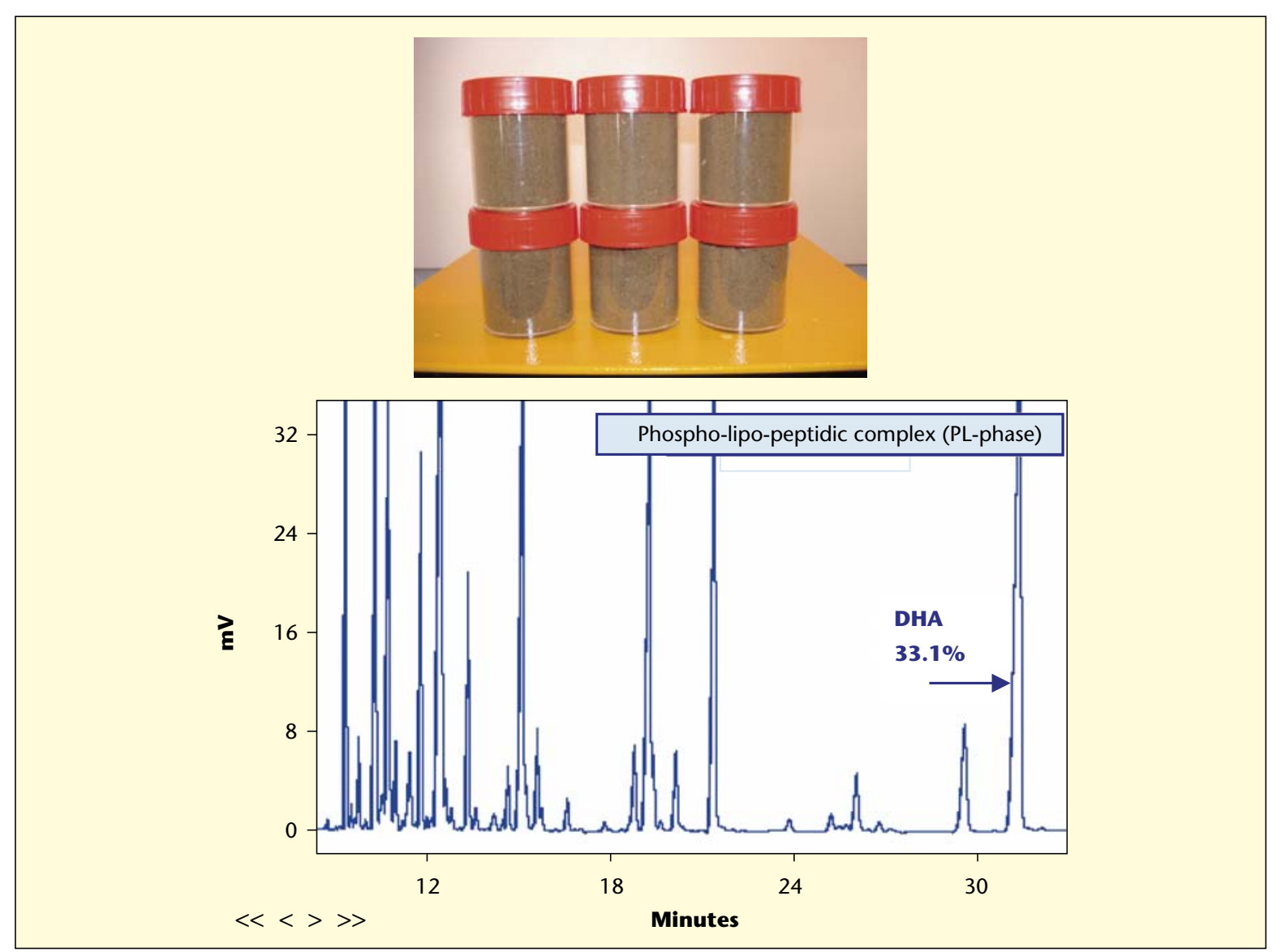

Figure 6. Fatty acid profile of the PL-phase of the phospho-lipo-peptidic complex.

ether/formic acid (80:20:0.2, v/v/v) shows that this complex contains $45 \%$ of TAG and $54 \%$ of PL (figure $7 A$ ). A second development using chloroform/methanol/ammonia (130:70:10, $\mathrm{v} / \mathrm{v} / \mathrm{v}$ ) allows the separation between PL molecules (figure 7B). Major PL is phosphatidylcholine (68.2\%), followed by phosphatidylethanolamine (10.8\%), phosphatidylserine (8.9\%), sphingomyeline (4.6\%) and phosphatidylinositol (4.8\%).

\section{Conclusion}

An enzymatic patented process has been validated at industrial scale to produce a phospholipo-peptidic complex extracted from salmon heads. The oily phase of this PLPC contains $54 \%$ of Phospholipids, which are particularly rich in DHA (more than 30\%). The final objective could be now to characterize how the product can affect the phospholipid composition of the cell-membrane with particular effect on the neuronal cell structure.

Acknowledgements. The authors are grateful to "Les Salaisons d'Orly" (SENIA, Rungis, France) and Laboratoires Le Stum, Lorient, France, for financial support. 

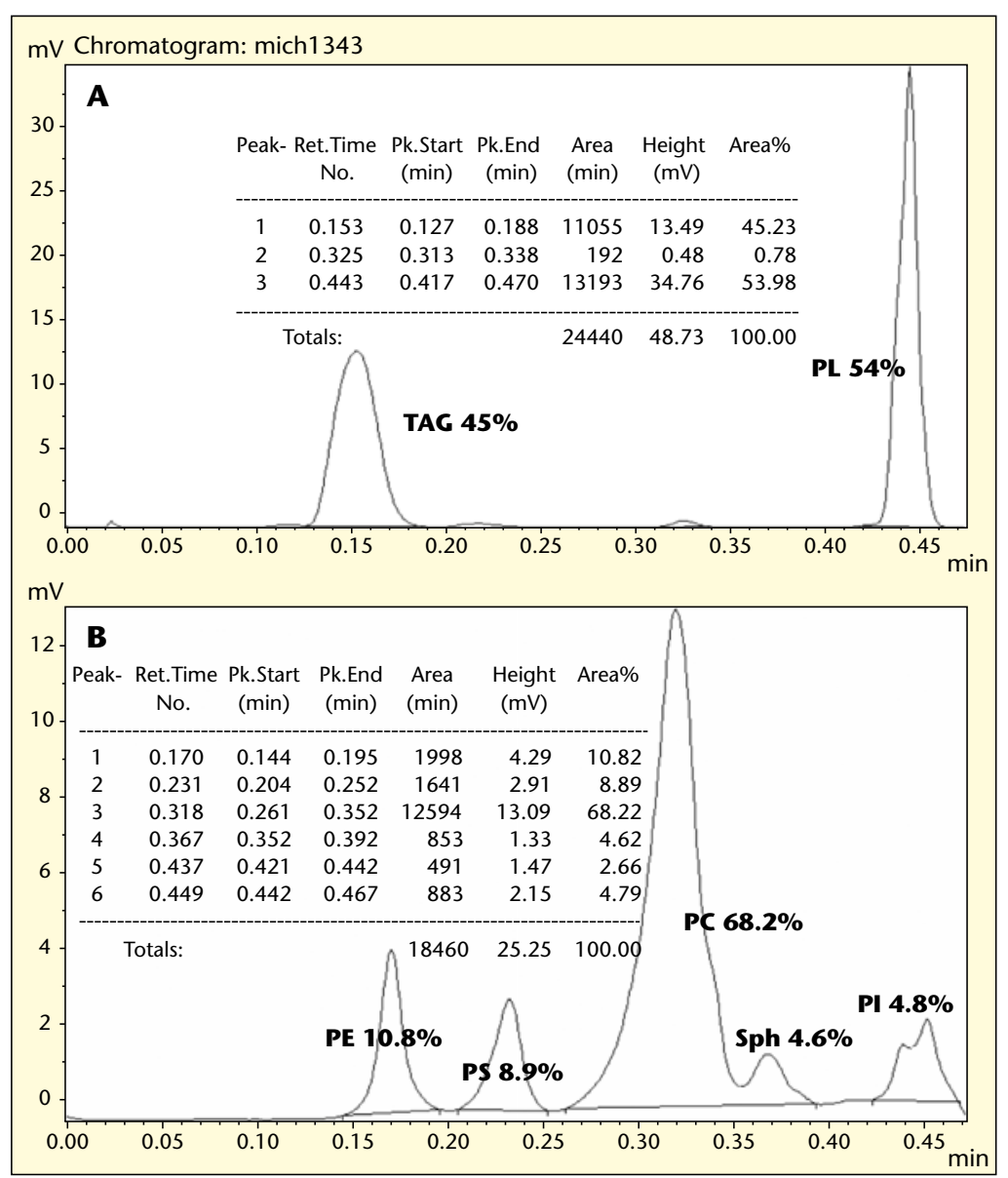

Figure 7. PLPC Lipid classes by TLC-FID.

A: $1^{\text {st }}$ developing tank: hexane/diethyl ether/formic acid (80:20:0. 2, v/v/v) to separate NL after 30 min. $B: 2^{\text {nd }}$ developing tank: chloroform/methanol/ammonia $(130: 70: 10, \mathrm{v} / \mathrm{v} / \mathrm{v})$ to separate PL after $40 \mathrm{~min}$.

\section{REFERENCES}

1. DUBOIS V, BRETON S, COMBE N, FANNI J, PARMENTIER M. FA composition of blood lipids, cholesterol esters and membrane phospholipids following a supplementation in marine LC-PUFA in rats, non published industrial results. Nice: Congrès francophone de nutrition, 2007.

2. SIMOPOULOS A. Omega-3 fatty acids in health and disease and in growth and development. Am / Clin Nutr 1991; 54: 438-63.

3. LEGRAND P. Comment augmenter l'apport nutritionnel en acides gras n-3. OCL 2004; 1(11): 50-4.

4. NAPIER J. LC-PUFA from plants, ELSA lecture, 4th EuroFedLipid congress, Madrid. 2006.

5. PENG J, LARONDELLEY, PHAMD, ACKMAN RG, ROLLIN X. Polyunsaturated fatty acid profiles of whole body phospholipids and triacylglycerols in anadromous and landlocked Atlantic salmon. Comparative Biochemistry and Physiology Part B 2003; 134: 335-48. Neurosci Lett 2002; 355: 129-33.
10. LAGARDE M, BERNOUD N, BROSSARD N. Lysophosphatidylcholine as a preferred carrier form of docosahexaenoic acid to the brain. I Mol Neurosci 2001; 16: 201-4.

11. ROTSTEIN NP, ILINCHETA DE BOSCHERO M, GIUSTO M, AVELDANO M. Effects of aging on the composition and metabolism of docosahexaenoate-containing lipids of retina. Lipids 22:253-60.

12. ANDO $S$, TANAKAY, TOYODA NEE OY, KON K, KAWASHIMA S. Turnover of synaptic membranes: age-related changes and modulation by dietary restriction. I Neurosci Res 2002; 70: 290-7.

13. BOURRE JM, PICIOTTI M. Delta-6 desaturation of alpha-linolenic acid in brain and liver during development and aging in the mouse. Neurosci Lett 1992; 141: 65-8.

14. KIDD PM. A review of nutrients and botanicals in the integrative management of cognitive dysfunction. Altern Med Rev 1999; 4: 144-61.

15. BERNOUD N, FENART L, MOLIERE $P$, et al. Preferential transfer of 2-docosahexaenoyl-1lysophosphatidylcholine through an in vitro blood-brain barrier over unesterified docosahexaenoic acid. J Neurochem 1999; 72: 338-45.

16. THIES F, PILLON C, MOLIERE P, LAGARDE M, LECERF J. Preferential incorporation of sn-2 lysoPC DHA over unesterified DHA in the young rat brain. Am I Physiol Regul Integr Comp Physiol 1994; 267: R1273-R1279.

17. WERNER A, HAVINGA R, KUIPERS F, VERKA$D E H J$. Treatment of EFA deficiency with dietary triglycerides or phospholipids in a murine model of extrahepatic cholestasis. Am / Physiol Gastrointest Liver Physiol 2004; 286: G822G832.

18. VAN ELSWYK M. Products containing highly unsaturated fatty acids for use by women and their children during stages of preconception, pregnancy and lactation/post-partum. US Patent WO 03/017945 A2. 2003.

19. HIRATSUKA S, TOSHIHIRO S, MASAYUKA H, et al. Method for extracting lipid mixture containing phospholipids comprising polyunsaturated fatty acids from viscera of fish, method for preserving viscera prior to extraction, and lipid mixture extracted thereby. US Patent 2003/0190392.

20. LEIGH S, KUNG E, VAN HOOGEVEST P, TIEMESSEN H. Marine lipid compositions, US Patent, WO 2004/047554 A1.

21. SAMPALIS F. Natural Marine source Phospholipids comprising Flavonoids, polyunsaturated fatty acids and their applications. WO/2003/011873.

9. BOURRE JM, DUMONT O. The administration of pig brain phospholipids versus soybean phospholipids in the diet during the period of brain development in the rat results in greater increments of brain docosahexaenoic acid.
22. LINDER M, FANNI J, PARMENTIER M, REGNAULT P. Procédé d'extraction d'huile par voie enzymatique et obtention d'hydrolysats protéiques à fonctionnalités dirigées. French Patent, 2. 835. 703. 2002. 